\title{
An URANS Simulation of the Kelvin-Helmholtz Aerodynamic Effect over the Ahmed Body
}

\author{
Sultan Sadykov ${ }^{1}$, Rinat Khalimov ${ }^{1}$, Yesitai Keleshibieke ${ }^{1}$, Sagidolla Batay ${ }^{1}$, Yong Zhao ${ }^{1 *}$ \\ 0000-0002-6693-2017, 0000-0003-2025-0034, 0000-0003-1673-5979, 0000-0001-8692-9571, 0000-0002-9574-4787
}

${ }^{1}$ Department of Mechanical Engineering, School of Engineering, Nazarbayev University, Astana, 010000, Republic of Kazakhstan

\section{Abstract}

The reduction of energy consumption of cars is always a significant issue in automotive design. Turbulent flow around a car body is very difficult to simulate accurately due to the complexity of the flow conditions around the body, such as complex flow separation and laminar to turbulent flow transition. In particular, flow over the Ahmed body with a rear angle of $25^{\circ}$ is considered a challenging problem for the RANS approach with two-equation turbulence models. In this study, we aim to analyze the Kelvin-Helmholtz instability associated with this flow with a URANS approach. Methodology for utilizing the URANS method is fully discussed. The predicted velocity profiles and drag coefficient are compared with experimental results. Three turbulence models, such as the k- $\varepsilon$, k- $\omega$ and SST models, are assessed and validated with experimental data. The aim of the study is to evaluate the performance of these models for the study of the Kelvin-Helmholtz instability over the Ahmed body and for car bodies generally using experimental data for their validations. It is found that the URANS approach with the turbulence models with proper numerical treatment can perform as well as or even better than the LES. And the SST model shows the best performance compared with other turbulence models.

Keywords: Ahmed body; Automotive aerodynamics; Car aerodynamic design; CFD; Kelvin- Helmholtz; LES; RANS; Turbulence models; URANS

\author{
Research Article \\ https://doi.org/10.30939/ijastech..923917
}

Received 25.04.2021

Revised 10.05.2021

Accepted 18.05.2021

* Corresponding author

Yong Zhao

yong.zhao@nu.edu.kz

Address: Mechanical Engineering Department, Nazarbayev University, Nur-Sultan, Kazakhstan

Tel: +77172704615

\section{Introduction}

The accepted paper must be prepared in two columns. The main text of the manuscript must be written in Times New Roman, font 10, 12-point line spacing. The font size, line spacing, and margin of the template must not be altered. Authors can use this template document to prepare the manuscript to submission. Authors can find and download this Microsoft Word document from the website of the journal, www.ijastech.org, Other submission versions will not be accepted, so, the manuscript could not go further to reviewing process.

The automobile is the most widespread form of transportation nowadays [1-2]. Fuel consumption is one of the important considerations in the designing of cars. Thus, it is important to optimize the aerodynamic shape of the car properly to minimize fuel consumption. Pressure drag and friction drag are the most important factors in aerodynamic design [3]. Aerodynamic drags can be minimized through geometry and flow modification of the vehicle since the geometry of the car is considered as one of the main factors affecting the drags [4].

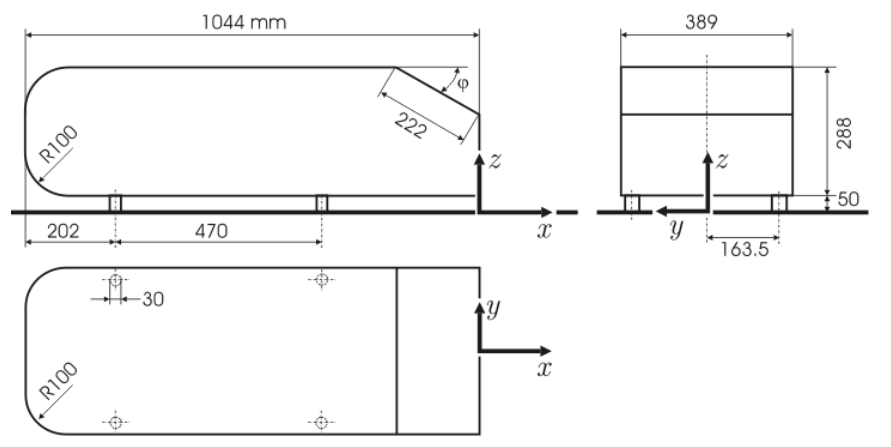

Fig. 1. Ahmed body

The Ahmed body has been used as a benchmark test case to validate numerical simulation models [5]. Nowadays, there are numerous methods of simulating airflow over cars, which include the Reynolds Averaged Navier Stokes (RANS) and Large Eddy Simulation (LES). The RANS approach is much more efficient than the LES one, but it can only simulate statistically steady flows. In this paper, the URANS method for unsteady turbulent flows will be investigated to find out if it is capable of modeling the Kelvin- 
Helmholtz effect found over Ahmed body with a slant angle of 25 degrees, which is said to be a challenging case for the RANS approach. Generally, it is believed that the LES method is more suitable for unsteady turbulent flow. However, the URANS approach with proper meshes and numerical methods can produce comparable results without using excessive computing resources, especially for engineers doing routine design evaluations [6]. In this, we study the URANS method and apply it to simulate the Kelvin-Helmholtz effect which cannot be modeled by the RANS approach and too time-consuming for the LES one [7-8]. The most important aspect in URANS simulation is to properly select the turbulence model and discretization scheme. It is noted that there is a second generation of URANS model, which is called the ScaleAdaptive Simulation and Partially Filtered Navier-Stokes [9]. However, in this paper, only the first generation model is considered [10]. The Kelvin-Helmholtz instability appears in the presence of a shear layer [11]. In the case of Kelvin-Helmholtz effect over the slant of the Ahmed body, it is caused by the separation and reattachment of the boundary layer over the slant. Different turbulence models will be tested in this project, such as k- $\omega, \mathrm{k}-\varepsilon$, and SST model. The $\mathrm{k}-\omega$ model can correctly predict the behavior of turbulence in the near wall region. However even if the turbulence model is not asymptotically consistent, skin friction and flow profile are predicted properly. In the wake region of the boundary layer, the k- $\omega$ model should be switched to the k- $\varepsilon$ model, which is less sensitive to the free stream turbulence boundary condition. When the flow separation occurs, it is better to utilize k- $\omega$ than k$\varepsilon$ [12]. The SST model combines $\mathrm{k}-\omega$ and $\mathrm{k}-\varepsilon$, automatically switching between the two models depending on the location in the boundary layer. Therefore, it is expected that the SST model will be able to model the boundary layer separation and reattachment over the slant better than other models.

\section{Methodology}

\section{1 Governing equations}

The Navier-Stokes equations for incompressible flow are

$\frac{\partial u_{i}}{\partial t}+\frac{\partial}{\partial x_{j}}\left[\begin{array}{ll}u_{i} & u_{j}\end{array}\right]=-\frac{1}{\rho} \frac{\partial P}{\partial x_{i}}+\frac{\partial \tau_{i j}}{\partial x_{j}}+\frac{\partial^{2} u_{i}}{\partial x^{2} k}$

k- $\omega$ model [12]

$$
\begin{aligned}
& \frac{\partial \rho k}{\partial t}=\tau_{i j} * \frac{\partial u_{i}}{\partial x_{j}}-\beta * \rho \omega k+\frac{\partial}{\partial x_{j}}\left[\left(\mu+\sigma_{k 1} * \mu_{1}\right) * \frac{\partial k}{\partial x_{j}}\right] \\
& \frac{D \rho \omega}{D t}=\frac{\gamma 1}{\vartheta t} * \tau_{i j} * \frac{\partial u_{i}}{\partial x_{j}}-\beta_{1} * \rho * \omega^{2}+\frac{\partial}{\partial x_{j}}\left[\left(\mu+\sigma_{w 1} * \mu_{t}\right) * \frac{\partial \omega}{\partial x_{j}}\right]
\end{aligned}
$$

Shear stress model:

$$
\begin{aligned}
& \frac{D \tau}{D t}=: \frac{d \tau}{d t}+u_{k} * \frac{d \tau}{d x_{k}} \\
& \tau=\rho * a 1 * k \\
& \tau=\mu_{t} * \frac{d u}{d y}
\end{aligned}
$$

$\tau=\rho * \sqrt{\frac{\text { production }}{\text { dissipation }}} * a_{1} * k$

Transformed k-e model:

$$
\begin{aligned}
& \frac{D \rho k}{D t}=\tau_{i j} * \frac{\partial u_{i}}{\partial x_{j}}-\beta * \rho * \omega * k+\frac{\partial}{\partial x_{j}}\left[\left(\mu+\sigma_{k 2} * \mu_{t}\right) * \frac{\partial k}{\partial x_{j}}\right] \\
& \frac{\mathrm{D} \rho \omega}{\mathrm{Dt}}=\frac{\gamma 2}{\vartheta \mathrm{t}} * \tau_{\mathrm{ij}} * \frac{\partial \mathrm{u}_{\mathrm{i}}}{\partial \mathrm{x}_{\mathrm{j}}}-\beta_{2} * \rho * \omega^{2}+\frac{\partial}{\partial \mathrm{x}_{\mathrm{j}}}\left[\left(\mu+\sigma_{\omega 2} * \mu_{\mathrm{t}}\right) * \frac{\partial \omega}{\partial \mathrm{x}_{\mathrm{j}}}\right]+ \\
& 2 \rho \sigma_{\omega 2} * \frac{1}{\omega} * \frac{\partial \mathrm{k}}{\partial \mathrm{x}_{\mathrm{j}}} * \frac{\partial \omega}{\partial \mathrm{x}_{\mathrm{j}}}
\end{aligned}
$$

Strouhal number

$S t=\frac{f H}{U}$

\section{Where}

f Frequency of oscillations

$\mathrm{H}$ Characteristic length of the Ahmed body

U Free stream velocity

$T$ Period of oscillations

$T_{\text {height }}$ Period of oscillations

$T_{\text {height }}$ Period of oscillations

At first, the height of the car was used as the characteristic length. The period of oscillations according to (1) becomes:

$$
\begin{gathered}
T_{\text {height }}=\frac{\text { height }}{S t * U}=\frac{0.288 \mathrm{~m}}{0.2 * 40 \mathrm{~m} / \mathrm{s}}=0.036 \mathrm{~s} \\
T_{\text {length }}=\frac{\text { length }}{S t * U}=\frac{1.044 \mathrm{~m}}{0.2 * 40 \mathrm{~m} / \mathrm{s}}=0.1305 \mathrm{~s}
\end{gathered}
$$

For transient simulation, timestep must be set up. To set up an initial guess for timestep, the following empirical formula was used:

$$
\Delta t=\frac{T_{\text {height }}}{10}=\frac{0.036}{10}=3.6 \mathrm{~ms}
$$

Where

\section{$\Delta \mathrm{t} \quad$ Transient simulation timestep}

However, this timestep was not small enough to converge to an oscillating solution. The Kevin-Helmholtz instability was not present. Moreover, the drag force was not oscillating, but converging to a certain value instead. For this reason, it was decided to decrease the timestep furthermore to $1 \mathrm{~ms}$. At timestep of $1 \mathrm{~ms}$, the simulation showed clear Kevin Helmholtz instability. The simulation results will be described below.

\subsection{Boundary conditions}

The simulation was performed with Ansys Fluent. The numerical model was established with dimensions described and boundary conditions given in Table 1 below. 
The dimensions of the Ahmed body are given below.

$\mathrm{W}(\mathrm{z}$-axis $)=389 \mathrm{~mm}$

$\mathrm{L}(\mathrm{x}$-axis $)=1044 \mathrm{~mm}$

$\mathrm{H}$ (y-axis) $=288 \mathrm{~mm}$

Table 1. Boundary and flow conditions

\begin{tabular}{c|c}
\hline Parameter names & Values and conditions \\
\hline Viscosity & $15 \times 10^{-6} \mathrm{~m}^{2} / \mathrm{s}$ \\
\hline Fluid & Air, $25^{\circ} \mathrm{C}$ \\
\hline $\begin{array}{c}\text { Reynolds number } \\
\text { Flow velocity at simulation domain } \\
\text { inlet }\end{array}$ & 768000 \\
\hline $\begin{array}{c}\text { Static gauge pressure at simulation } \\
\text { domain outlet }\end{array}$ & 0 Pa $/ \mathrm{s}$ \\
\hline Car body and ground & No slip wall \\
\hline $\begin{array}{c}\text { The top boundary of the simulation do- } \\
\text { main }\end{array}$ & Free slip wall \\
\hline
\end{tabular}

\subsection{Mesh convergence study}

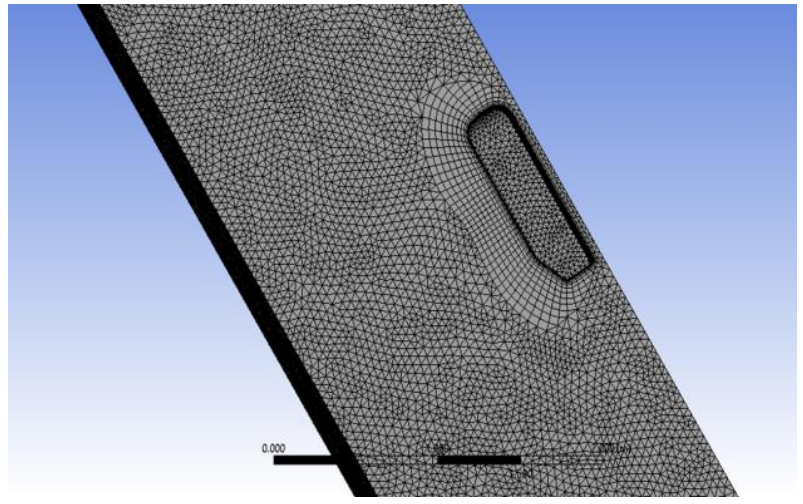

Fig. 2. Initial mesh

Figure 2 shows the initial mesh. Proper meshing is one of the most important factors in CFD simulations. To improve the accuracy of the simulation, the mesh near the car surface was refined with the help of the inflation layer. The structured mesh was used near the surfaces because of turbulent boundary layers.

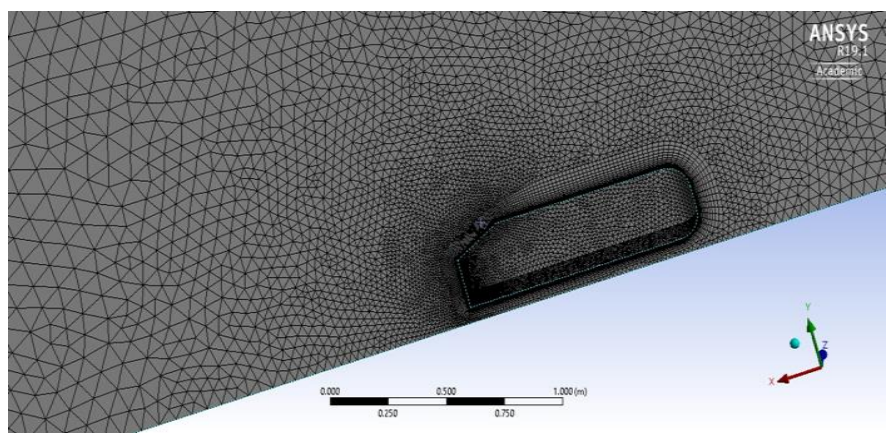

Fig 3. Finest mesh

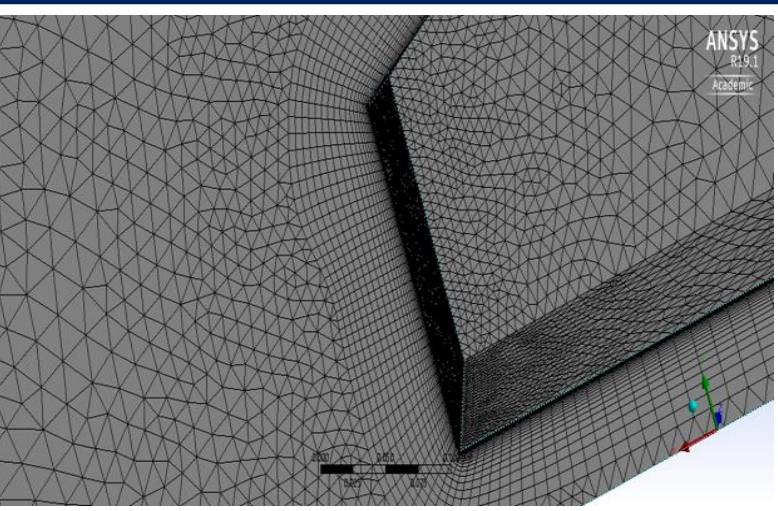

Fig. 4. Fine mesh

The finest and fine meshes are shown in Figures 3 and 4 respectively. A mesh convergence study was carried out before the relative variations of key parameters were less than $1 \%$. Five different meshes were selected. The variations of velocity, drag coefficient and pressure with mesh density at selected locations were examined, as shown in Table 2.

Table 2. Mesh convergence

\begin{tabular}{c|c|c|c|c}
\hline Mesh No. & $\begin{array}{c}\text { No of } \\
\text { elements }\end{array}$ & $\begin{array}{c}\text { Drag } \\
\text { coefficient }\end{array}$ & $\begin{array}{c}\text { Velocity } \\
(\mathrm{m} / \mathrm{s})\end{array}$ & Pressure (Pa) \\
\hline 1 & 423540 & 0,342 & 23,14 & $-1481,52$ \\
\hline 2 & 605349 & 0,32 & 23,77 & $-1481,90$ \\
\hline 3 & 875843 & 0,314 & 22,80 & $-1468,23$ \\
\hline 4 & 987653 & 0,310 & 23,04 & $-1434,39$ \\
\hline 5 & 1234053 & 0,293 & 22,82 & $-1453,52$ \\
\hline
\end{tabular}

From Table 2 the optimal mesh was found to be mesh number 5 , which has 1,234,053 elements and produced overall best results as shown in Table 4.

\subsection{Validation study}

Table 3. Probe locations

\begin{tabular}{c|c|c|c}
\hline Probe \# & $\mathrm{X}(\mathrm{mm})$ & $\mathrm{Y}(\mathrm{mm})$ & $\mathrm{Z}(\mathrm{mm})$ \\
\hline 1 & 80 & 338 & 100 \\
\hline 2 & 80 & 28 & 100 \\
\hline 3 & 500 & 178 & 160 \\
\hline 4 & 1062 & 148 & 0 \\
\hline 5 & 1442 & 28 & 0 \\
\hline
\end{tabular}

Table 3 shows the coordinates of the probes.

Table 4. The error of drag, velocity, and pressure

\begin{tabular}{c|c|c|c}
\hline Mesh \# & $\begin{array}{c}\text { Errors of } \\
\text { drag }(\%)\end{array}$ & $\begin{array}{c}\text { Max. Errors } \\
\text { of Velocity }\end{array}$ & $\begin{array}{c}\text { Max. Er- } \\
\text { rors of }\end{array}$ \\
\hline 1 & 6.9 & 8.05 & 0.95 \\
\hline 2 & 3.90 & 2.32 & 0.88 \\
\hline 3 & 2.53 & 4.01 & 0.97 \\
\hline 4 & 1.05 & 2.55 & 0.53 \\
\hline 5 & 1.68 & 1.55 & 0.67 \\
\hline
\end{tabular}


Table 5. Comparison of velocity predicted by different turbulence

\begin{tabular}{c|c|c|c}
\hline \multicolumn{4}{|c}{ models } \\
\hline Probe \# & SST (\%) & $\mathrm{k}-\varepsilon(\%)$ & $\mathrm{k}-\omega(\%)$ \\
\hline 1 & 1.519 & 3.040 & 1.313 \\
\hline 2 & 12.259 & 15.904 & 14.896 \\
\hline 3 & 4.824 & 4.511 & 4.413 \\
\hline 4 & 4.682 & 6.127 & 10.071 \\
\hline 5 & 1.747 & 3.849 & 0.36 \\
\hline
\end{tabular}

Table 5 shows errors in velocity for different turbulence models.

\begin{tabular}{c|c|c}
\hline Turbulence models & $\begin{array}{c}\text { Drag } \\
\text { coefficient }\end{array}$ & Drag coefficient error (\%) \\
\hline SST & 0.303 & 1.68 \\
\hline k- $\varepsilon$ & 0.3156 & 5.91 \\
\hline k- $\omega$ & 0.335 & 12.4 \\
\hline $\begin{array}{c}\text { Experimental Data } \\
\text { (Ahmed et. al., 1984) }\end{array}$ & 0.298 & NA \\
\hline
\end{tabular}

Table 6. Drag coefficient predicted by various turbulence models.

Table 6 shows obtained drag coefficient values for different turbulence models. As shown in the table, drag coefficient with SST model gave the closest results to experimental values.

\section{Results and Discussion}

Figures 5 and 6 show velocity and pressure contours respectively for steady-state RANS simulation. As can be noticed, there are no large scale eddies formed, which is caused by the reason that Ansys Fluent solver tries to converge on solution where velocity values for every nodes do not oscillate and approach final static solution. It can be clearly seen that in 25 degree slant angle, the flow starts to separate before meeting slanted surface. Since the flow separation occurs very soon, there is a pressure increase due to large separation region, which leads to an increase in drag coefficient.

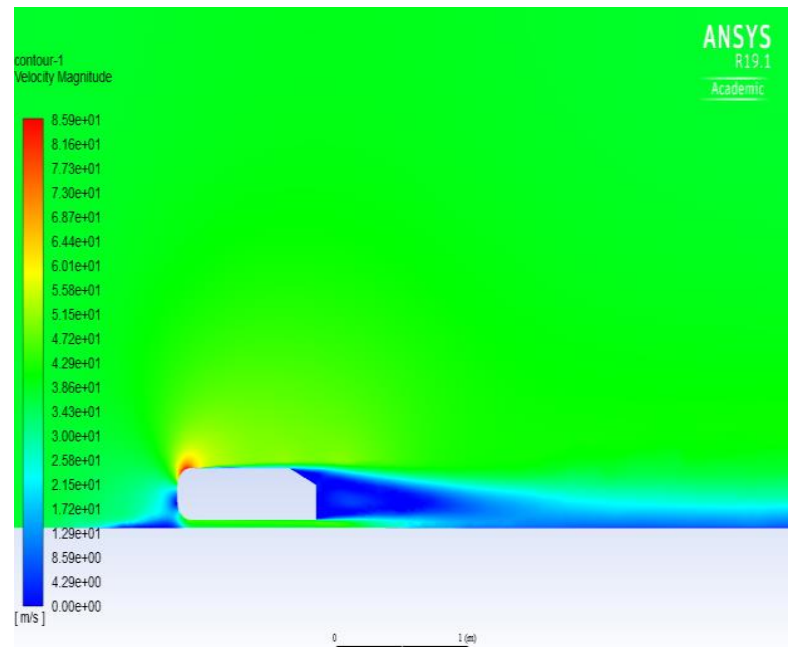

Fig. 5. Velocity contour for RANS simulation equation was applied, and streamlines that passes through the inlet and stagnates into the front of the car. It can be stated that at the first point, the velocity is $40 \mathrm{~m} / \mathrm{s}$ at the inlet, and at the second point the velocity is zero, which means that the kinematic energy of the fluid fully transforms into static pressure. In other words, the static pressure at the front of the car must rise. As can be seen in Figure 6 , the pressure at the front of the car is $1220 \mathrm{~Pa}$ higher than atmospheric pressure, which agrees with the aforementioned theory. According to pressure contour, the pressure at the inlet boundary near the Ahmed body is $280 \mathrm{~Pa}$. Therefore, the dynamic pressure is

$$
\mathrm{P}_{\text {dynamic }}=\frac{\rho \mathrm{u}^{2}}{2}=1220-280=940 \mathrm{~Pa}
$$

The free stream velocity can be extracted from the aforementioned formula:

$$
\mathrm{u}=\sqrt{\frac{2 \mathrm{P}_{\text {dynamic }}}{\rho}}=\sqrt{\frac{2 * 940}{1.225}}=39.18 \mathrm{~m} / \mathrm{s}
$$

The actual free stream velocity that is set up in Ansys Fluent solver is $40 \mathrm{~m} / \mathrm{s}$, which is close to the aforementioned guess.

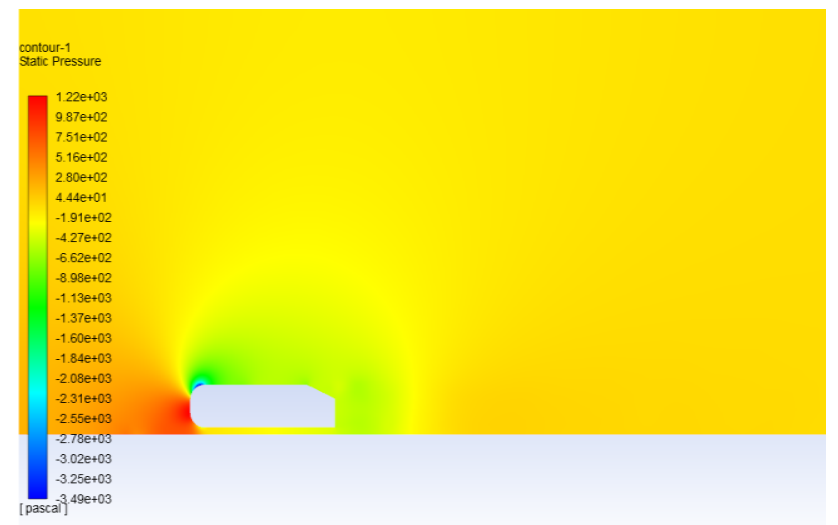

Fig. 6. Pressure contour for RANS simulation

Velocity contour shown below in figure 7 for unsteady simulation differs significantly with steady-state simulation. This is because the RANS simulation tends to find optimal velocity distribution configuration, such that, there is no alteration with time. Whereas, in transient simulation, the flow changes after each timestep. In other words, in transient simulation, the flow can be either steady or unsteady. Whereas in steady simulation, the flow will be steady, otherwise, the solution will not converge. Figure 7 below shows clear large eddy formation, unlike in Figure 5. The eddies move in the right direction. The eddies are created with a constant frequency. It was found that the period of oscillations is $0.029 \mathrm{~s}$.

Figure 6 demonstrates the pressure distribution. Bernoulli's 


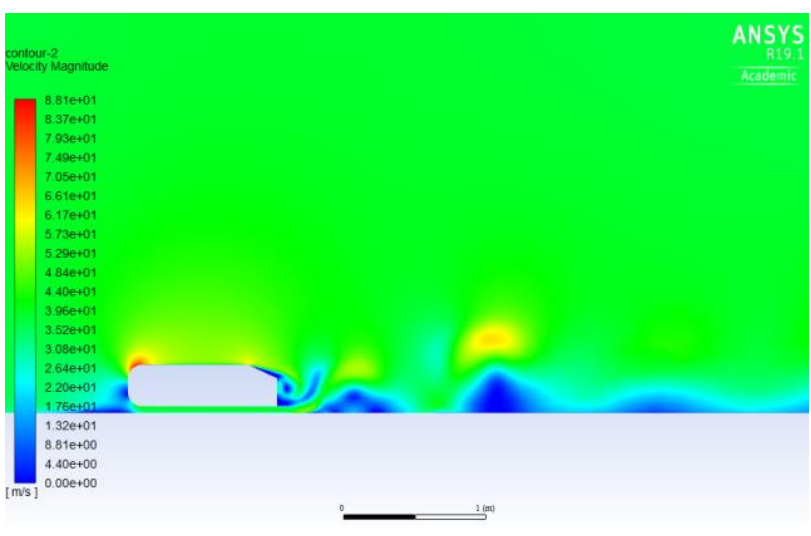

Fig. 7. Velocity contour for URANS simulation

Similarly, as velocity contour in URANS simulation, the pressure contour does not match the RANS simulations. (figure 8) There is a clear formation of large-scaled eddies. Unlike in steadystate simulation (Figure 6), there is a pressure gradient in every eddy formation. The gauge pressure inside an eddy is negative, whereas the pressure outside and near this eddy is positive. The URANS provides a more accurate representation of real flow if compared to RANS. This is because the behavior of real-world fluids is always unsteady in turbulent flows, and RANS was developed to decrease computational time.

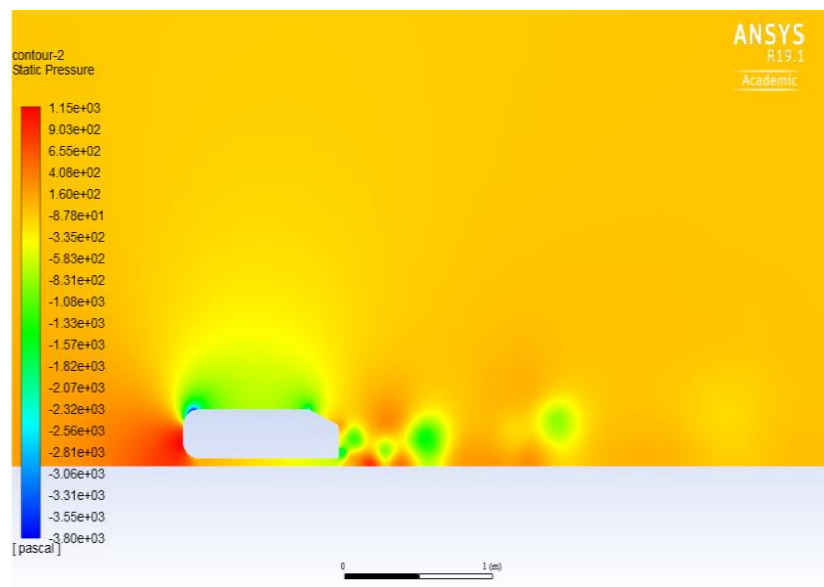

Fig. 8. Pressure contour for URANS simulation.

During the simulation, the drag coefficient was computed in each timestep. In result, Figure 9 shows a chart of drag coefficient vs time of flow. At time $=0 \mathrm{~s}$, the flow was initialized with Hybrid initialization, giving an initial approximation of pressure and velocity distributions. It is possible to initialize the flow with zero velocity at every mesh node, however, using this simple method, the flow will converge slower to a final solution. Whereas, with Hybrid initialization, the flow converges faster, resulting in lower computational time. It can be seen on the chart that during the first $0.2 \mathrm{~s}$, the drag coefficient is changing with time, but the flow is not stable. After $0.2 \mathrm{~s}$, the flow still varies with time but does not vary across every period. To calculate the period of oscillations, a snapshot of flow at $0.2 \mathrm{~s}$ was taken, later each frame was analyzed. Following frames were compared to the first snapshot frame at $0.2 \mathrm{~s}$. In results, after each $0.029 \mathrm{~s}$ the velocity profile exactly matches the snapshot frame. Which means that the period of oscillations is $0.029 \mathrm{~s}$.

As has been mentioned earlier, the initial guesses for the period of oscillations according to Strouhal's number are $T_{\text {height }}=$ $0.036 \mathrm{~s}$ and $T_{\text {height }}=0.1305 \mathrm{~s}$, which is a good initial guess for setting up timestep value. Since the period of oscillations is known at this point, the actual Strouhal's number can be calculated using formula as follows:

$$
\mathrm{St}=\mathrm{fL} / \mathrm{U}=\mathrm{L} /(\mathrm{TU})=0.288 /(0.029 * 40)=0.248
$$

Since the period of oscillations according to simulation is 0.029 , it can be concluded that the Strouhal's number should be calculated with the height of the car as characteristic length instead of the car's length.

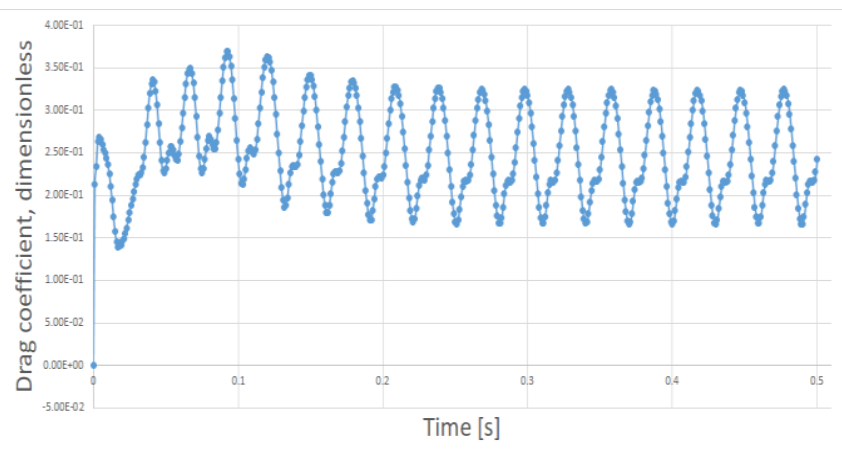

Fig. 9. Drag coefficient varies over time.

The $\mathrm{x}$-axis represents time, Y-axis represents the drag coefficient.

Figure 10 below shows experimental velocity profile values obtained from [5] shown with round points. The simulation velocity profile data is shown with triangular points. As can be noticed the simulated velocity profile agrees with experimental values as expected. Experimental data were taken from [13].

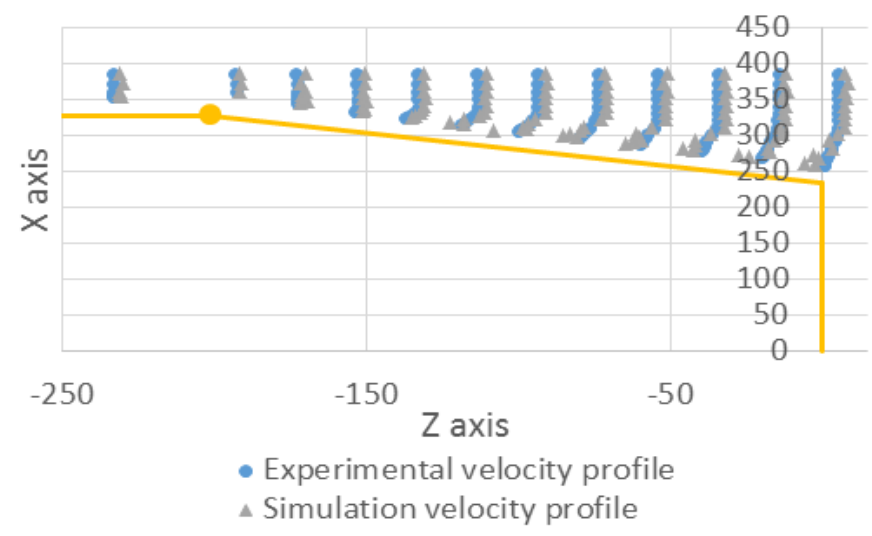

Fig. 10. Comparison of experimental and simulated velocity profiles. 


\section{Conclusion}

This paper presents a detailed study on the use of the URANS method with three turbulence models for simulating unsteady turbulent separating flow over the Ahmed body with a rear angle of 25 degrees with the Kelvin-Helmholtz effect. When an optimal mesh was developed and adopted with a proper discretizing scheme, the URANS method performed as well as LES models, or even better in some cases. The results produced by the URANS method with three different turbulence models showed good agreement with experimental data and the SST model produced the best results, with only $1.68 \%$ in discrepancy for the predicted drag and $1.3 \%$ for predicted velocity. Generally, the URANS is found to be a reliable and accurate tool for the prediction of unsteady separating turbulent airflow over the Ahmed body. It is believed that outcomes from this work could help automotive designers to enhance design productivity.

\section{Acknowledgement}

This study is funded by Nazarbayev University through a FDCR grant No. 240919FD3934

\begin{tabular}{ll}
\multicolumn{2}{l}{ Nomenclature } \\
$\rho:$ & density, $\mathrm{kg} / \mathrm{m}^{3}$ \\
$\mathrm{u}:$ & velocity, $\mathrm{m} / \mathrm{s}$ \\
$\tau:$ & stress tensor, $\mathrm{N} / \mathrm{m}^{2}$ \\
$\mu:$ & fluid dynamic viscosity, Pas \\
$\delta:$ & the Kronecker delta \\
$\mathrm{x}:$ & Cartesian coordinates \\
$\mathrm{k}:$ & turbulence kinetic energy per unit mass, $\mathrm{j} / \mathrm{kg}$ \\
$\varepsilon:$ & turbulence dissipation rate per unit mass, $\mathrm{j} /(\mathrm{kg} \cdot \mathrm{s})$ \\
$\omega:$ & rate of dissipation of eddies, $\mathrm{j} /(\mathrm{kg} \cdot \mathrm{s})$ \\
$v:$ & kinematic viscosity, $\mathrm{m}^{2} / \mathrm{s}$ \\
$\Omega:$ & absolute value of vorticity, $1 / \mathrm{s}$ \\
$\mathrm{C}_{\mathrm{D}}:$ & drag coefficient, dimensionless
\end{tabular}

\section{Subscripts}

$\begin{array}{ll}\text { i,j: } & \text { Cartesian coordinate indexes } \\ \text { t: } & \text { turbulence }\end{array}$

\section{Conflict of Interest Statement}

There is no conflict of interest in the study.

\section{CRediT Author Statement}

Yong Zhao: Conceptualization, Supervision, Funding acquisition, Project administration, Resources

Sultan Sadykov: Conceptualization, Writing-original draft, Validation, Methodology, Data curation, Visualization, Investigation
Rinat Khalimov: Conceptualization, Writing-original draft, Validation, Software, Visualization, Investigation

Yesitai Keleshibieke: Formal analysis, Validation

Sagidolla Batay: Formal analysis, Writing - review \& editing

\section{References}

[1] Menter F. Two-equation eddy-viscosity turbulence models for engineering applications. AIAA Journal. 1994;32(8):1598-1605.

[2] Serre E, Minguez M, Pasquetti R, Guilmineau E, Deng G, Kornhaas $\mathrm{M}$ et al. On simulating the turbulent flow around the Ahmed body: A French-German collaborative evaluation of LES and DES. Computers \& Fluids. 2013;78:10-23.

[3] Igali, D., Mukhmetov, O., Zhao, Y., Fok, S. and Teh, S. Comparative Analysis of Turbulence Models for Automotive Aerodynamic Simulation and Design. International Journal of Automotive Technology. 2019;20(6):1145-1152.

[4] Marklund A. EDITORIAL. Journal of Scandinavian Cinema. 2013;3(1):3-5.

[5] Ahmed, S R, Ramm, G, and Faitin, G. Some salient features of the time - averaged ground vehicle wake. United States: N. p., 1984. Web.

[6] Léonard T, Gicquel L, Gourdain N, Duchaine F. Steady/Unsteady Reynolds-Averaged Navier-Stokes and Large Eddy Simulations of a Turbine Blade at High Subsonic Outlet Mach Number. Journal of Turbomachinery. 2014;137(4).

[7] Bayraktar I, Landman D, Baysal O. Experimental and Computational Investigation of Ahmed Body for Ground Vehicle Aerodynamics. SAE Technical Paper Series. 2001.

[8] Gilliéron P, Chometon F. Modelling of stationary three-dimensional separated air flows around an Ahmed reference model. ESAIM: Proceedings. 1999;7:173-182.

[9] ROSHKO, A. On the Wake and Drag of Bluff Bodies. Journal of the Aeronautical Sciences. 1955;22(2):124-132.

[10]Argyropoulos C, Markatos N. Recent advances on the numerical modelling of turbulent flows. Applied Mathematical Modelling. 2015;39(2):693-732.

[11]Drazin P. Meteorology and numerical analysis. Cambridge: Cambridge University Press; 1993.

[12]Schwarze R, Obermeier F. Performance and limitations of the unsteady RANS approach. PAMM. 2006;6(1):543-544.

[13]Welcome to cfd.mace.manchester.ac.uk [Internet]. Cfd.mace.manchester.ac.uk. 2021 [cited 22 May 2021]. Available from: http://cfd.mace.manchester.ac.uk/ 